\title{
An adaptive scheme for diffuse- optical tomography based on combined structured-light illumination and single-pixel camera detection
}

Farina, Andrea, Betcke, Marta, Bassi, Andrea, Valentini, Gianluca, Arridge, Simon, et al.

Andrea Farina, Marta Betcke, Andrea Bassi, Gianluca Valentini, Simon Arridge, Cosimo D'Andrea, "An adaptive scheme for diffuse-optical tomography based on combined structured-light illumination and single-pixel camera detection," Proc. SPIE 11074, Diffuse Optical Spectroscopy and Imaging VII, 110742I (11 July 2019); doi: 10.1117/12.2527689 


\title{
An adaptive scheme for diffuse-optical tomography, based on combined structured-light illumination and single-pixel camera detection
}

\author{
Andrea Farina $^{\mathrm{a}}$, Marta Betcke ${ }^{\mathrm{b}}$, Andrea Bassi ${ }^{\mathrm{a}, \mathrm{c}}$, Gianluca Valentini ${ }^{\mathrm{a}, \mathrm{c}}$, Simon Arridge ${ }^{\mathrm{b}}$, and \\ Cosimo D'Andrea ${ }^{\mathrm{c}, \mathrm{d}}$
}

${ }^{a}$ Consiglio Nazionale delle Ricerche, Istituto di Fotonica e Nanotecnologie, Piazza Leonardo da Vinci 32, 20133, Milano, Italy

${ }^{\mathrm{b}}$ Centre for Medical Image Computing, University College London, Malet Place, London WC1E 6BT, UK

'Politecnico di Milano, Dipartimento di Fisica, Piazza L. da Vinci 32, 20133 Milano, Italy

${ }^{\mathrm{d}}$ Center for Nano Science and Technology@PoliMi, Istituto Italiano di Tecnologia, 20133 Milan, Italy

\begin{abstract}
Diffuse Optical Tomography (DOT) is a tool for 3D reconstruction of absorption and scattering inside a tissue. Typically, this method requires a dense distribution of sources and detectors, thus hampering the possibility of fully exploring a time-resolved detection. Recently, techniques based on structured-light illumination and compressing detection have been developed, opening the possibility of fully exploiting a source/detector spatial modulation for compression at the measurement stage. Here we propose a combined Continuous-Wave (CW) and time-domain (TD) adaptive scheme based on the singular-value decomposition (SVD) for optimal-patterns calculation. Patterns are firstly computed based on a fast acquisition via a CCD, and consequently projected for time-resolved measurements.
\end{abstract}

Keywords: Diffuse Optical Tomography, structured light, adaptive algorithm, time-resolved, compressive sensing, singular-value decomposition

\section{INTRODUCTION}

Diffuse Optical Tomography (DOT) is a biomedical imaging tool capable of reconstructing a three-dimensional map of the optical properties (absorption and scattering coefficients) from measurements taken on the boundary of the tissue. The classical approach to DOT is based on a dense coverage of source/detector pairs placed on the surface of the sample. ${ }^{1}$ This dense coverage is usually achieved using point-like sources and singleelement detector, making the system quite complex and expensive, in particular if time-resolved detection is needed for increasing penetration depth or contemporary discrimination of absorption and scattering. The contemporary implementation of a high-density coverage of source/detector pairs and time-resolved detection is very challenging, so compression strategy are strongly required. A possibility to overcome this limitation is the use of structured light illumination and compressive detection.

In the recent years there have been an increasing attention to adaptive algorithms in which measurement results drive new acquisitions strategies in real-time. ${ }^{2-4}$ This is important for reducing both acquisition and computation times. In the field of DOT, this can be achieved using a wide-field approach both in the input and output sides. In this work, we propose a combined CW - TD compression scheme capable of allowing optimal pattern computation after a fast widefield CW acquisition. Once patterns have been selected, a small amount of TD measurements has been performed.

Further author information: (Send correspondence to Andrea Farina)

Andrea Farina: E-mail: andrea.farina@polimi.it

Diffuse Optical Spectroscopy and Imaging VII, edited by Hamid Dehghani, Heidrun Wabnitz, Proc. of SPIE-OSA Vol. 11074, 110742I · C 2019 SPIE-OSA · CCC code: 1605-7422/19/\$21 · doi: 10.1117/12.2527689 


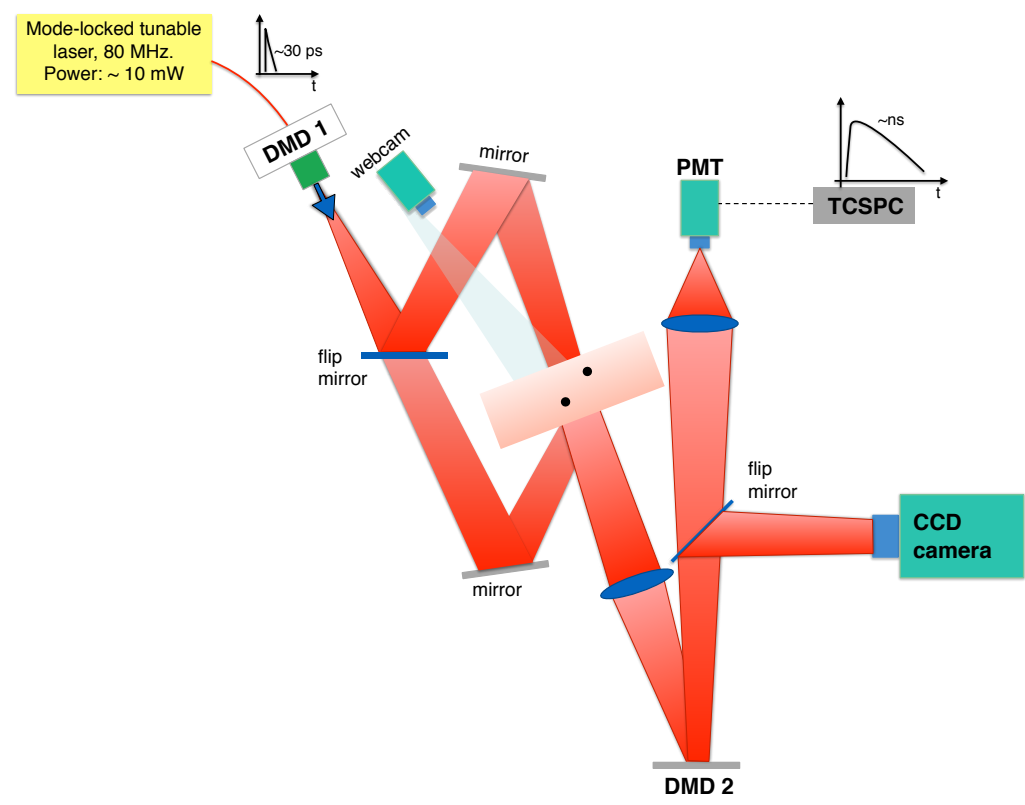

Figure 1. Experimental setup. In the figure the direction of light is depicted in red. After the first DMD (DMD 1), patterned light is projected on the sample either in reflection or in transmission geometry by means of a flip mirror. On the detection side, after the second DMD (DMD 2), the light can either be focused on a Photomultiplier Tube (PMT) for time-resolved detection or on a CCD camera, by means of a second flip mirror.

\section{MATERIAL AND METHODS}

The setup is depicted in Fig.1. A supercontinuum fiber laser source (SC-450, Fianium) provides mode-locking pulses $(40 \mathrm{MHz})$ in the spectral range $450-1750 \mathrm{~nm}$. The collimated output first passes through a short-pass

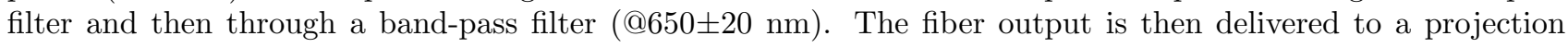
system coupled to a Digital Micromirror Device (DMD) for patterned illumination. After the projection system, an achromatic lens $(\mathrm{f}=75 \mathrm{~mm})$ projects patterns on the input plane of the sample. This is achieved by means of a flip mirror and a second mirror for the capability of working both in reflectance and transmittance modalities. The output plane of the sample is then imaged on a second DMD device for implementation of the single-pixel camera. The DMD plane is then optically conjugated to either a cooled CCD or to a step-index optical fiber tip through a long-working distance objective lens (10x). The two paths can be switched by means of a flip mirror. The step-index fiber is finally connected to an imaging system based on two achromatic lenses for coupling the light to a photomultiplier tube (PMT). This PMT is connected to a Time-Correlated Single-Photon Counting (TCSPC) board for time-resolved acquisition. The experiment have been performed on a slab phantom $(15 \times 64$ $\mathrm{x} 64 \mathrm{~mm}^{3}$ ) with background optical properties of $\mu_{a}=0.01 \mathrm{~mm}^{-1}, \mu_{s}^{\prime}=1 \mathrm{~mm}^{-1}$ with two holes drilled at slightly different depths with respect to the two squared surfaces. A cylindrical black rod has been placed in the holes alternatively on the input and output sides.

To find optimal patterns both for the input and the output side, Singular-Value Decomposition (SVD) has been applied. In particular, a contrast matrix $A$ has been constructed with a sequence of differential CCD images (with and without perturbation) acquired when a point illumination on the input side raster scans the illumination area. It is worth noting that the input pattern set can be any basis, such as Walsh-Hadamard, Wavelet, Fourier. By performing SVD of the matrix $A$ it is possible to retrieve a set of input $\left(\mathbf{v}_{i}\right)$ and output $\left(\mathbf{u}_{i}\right)$ vectors (i.e. patterns) ordered by the singular-values $\lambda_{i}$ that, in turns, give the respective weight of the two i-th input/output vectors. In particular the decomposition is as follow:

$$
A=U \Lambda V^{T}
$$

where the columns of $\mathrm{U}$ are $\mathbf{u}_{i}$, the columns of $\mathrm{V}$ are $\mathbf{v}_{i}$ and $\Lambda$ is a diagonal matrix containing the singular-values $\lambda_{i}$ ordered by their magnitude. Due to magnitude-ordered characteristic of the singular values $\lambda_{i}$, it is possible 
Recon Mua
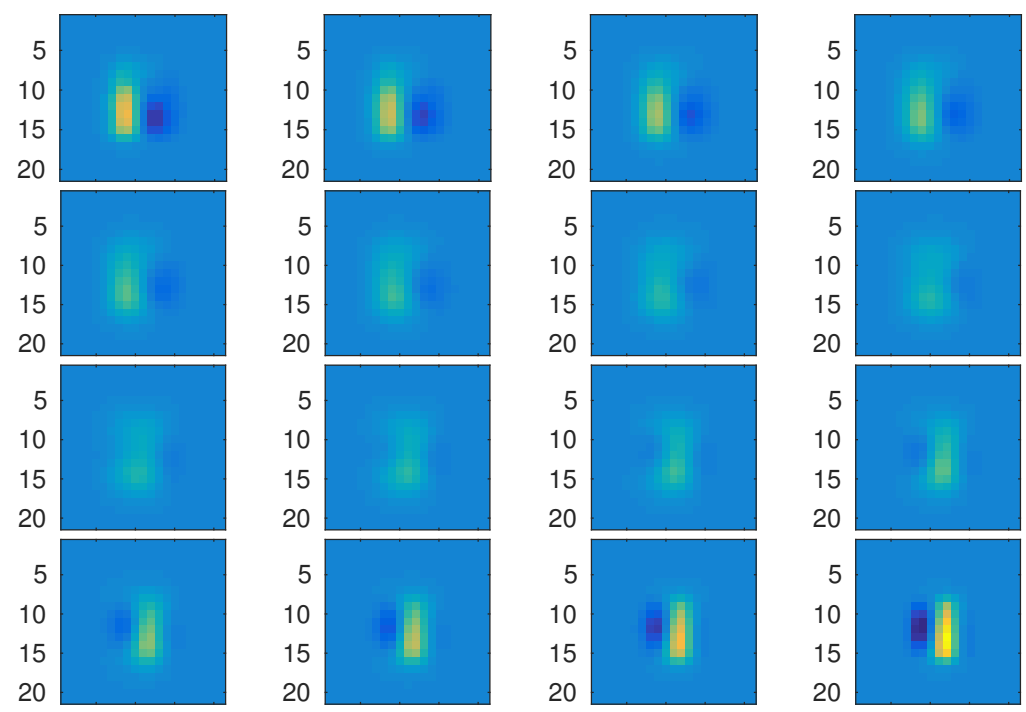

Figure 2. Reconstruction of two perturbations drilled in the slabs.

to truncate the spectrum to a certain order $k$ and reconstruct an approximated version $A_{k}$ given by:

$$
A_{k}=U \Lambda_{k} V^{T}
$$

where $\Lambda_{k}$ is the reduced singular-values diagonal matrix. It is worth noting that no positivity constrain in the SVD is imposed, thus for every pattern two positive patterns were implemented on the DMDs after proper area normalization. A truncation to the fifth order is enough for good reconstruction. Preliminary reconstructions have been obtained using (see Fig. 2) under the Born approximation. A finite-element based forward solver $(\mathrm{TOAST}++)^{5}$ has been used for the modelling of the propagation of light through the diffusive slab.

\section{CONCLUSIONS}

In this work, we have devised a combined CW - TD methods for optimal pattern generation. Optimal patterns have been retrieved through a fast CW acquisition, afterwrds a subset have been selected and then projected back for time-resolved detection.

\section{REFERENCES}

[1] Eggebrecht, A. T., Ferradal, S. L., Robichaux-Viehoever, A., Hassanpour, M. S., Dehghani, H., Snyder, A. Z., Hershey, T., and Culver, J. P., "Mapping distributed brain function and networks with diffuse optical tomography," Nature Photonics 8(6), 448-454 (2014).

[2] Soldevila, F., Salvador-Balaguer, E., Clemente, P., Tajahuerce, E., and Lancis, J., "High-resolution adaptive imaging with a single photodiode," Scientific reports 5, 14300 (2015).

[3] Tian, L., Liu, Z., Yeh, L.-H., Chen, M., Zhong, J., and Waller, L., "Computational illumination for high-speed in vitro Fourier ptychographic microscopy," Optica 2(10) (2015).

[4] Rousset, F., Ducros, N., Peyrin, F., Valentini, G., DAndrea, C., Farina, A., D'Andrea, C., and Farina, A., "Time-resolved multispectral imaging based on an adaptive single-pixel camera," Optics Express 26(8), 10550-10558 (2018).

[5] Schweiger, M. and Arridge, S., "The Toast ++ software suite for forward and inverse modeling in optical tomography," Journal of biomedical optics 19(4), 1-15 (2014). 